\title{
Polysèmes
}

Revue d'études intertextuelles et intermédiales

\section{La subversion du descriptif dans les romans d'Elizabeth Bowen}

Shannon Wells-Lassagne

\section{(2) OpenEdition}

Journals

Édition électronique

URL : http://journals.openedition.org/polysemes/1752

DOI : $10.4000 /$ polysemes. 1752

ISSN : 2496-4212

Éditeur

SAIT

Édition imprimée

Date de publication : 2 janvier 2007

Pagination : 73-91

ISSN : 0999-4203

\section{Référence électronique}

Shannon Wells-Lassagne, «La subversion du descriptif dans les romans d'Elizabeth Bowen »,

Polysèmes [En ligne], 9| 2007, mis en ligne le 01 janvier 2007, consulté le 19 avril 2019. URL : http:// journals.openedition.org/polysemes/1752 ; DOI : 10.4000/polysemes.1752

Ce document a été généré automatiquement le 19 avril 2019.

Polysèmes 


\title{
La subversion du descriptif dans les romans d'Elizabeth Bowen
}

\author{
Shannon Wells-Lassagne
}

Philippe Hamon, dans l'introduction de son ouvrage sur le descriptif, rappelle le peu de considération généralement accordé à la description ${ }^{1}$. En effet, elle est souvent perçue comme superflue, comme un temps d'arrêt que marque l'auteur avant de revenir à l'essentiel, à savoir «l'action » du roman. Toutefois, malgré son caractère en apparence interstitiel, la description peut aussi être le lieu d'enjeux bien plus importants pour le roman que l'on pourrait le croire de prime abord. Ainsi, dans l'œuvre d'Elizabeth Bowen, la description est loin d'être accessoire: elle constitue un moment d'une complexité particulière, dans laquelle se dessinent des éléments essentiels, et pour «l'action» du roman qui l'entoure, et pour l'art romanesque de l'auteur. Cette complexité se joue en particulier dans sa nature éminemment subversive, qui traverse tous les romans de Bowen.

2 Mettre en rapport la subversion et la description peut sembler redondant, voire tautologique. Comme l'écrit Hamon, l'essence même du descriptif est de subvertir la linéarité du mode narratif qui prévaut dans le roman classique pour arrêter l'action, en modifiant la structure chronologique du récit qui devient alors beaucoup plus complexe ${ }^{2}$. Ce type de subversion se manifeste évidemment dans l'œuvre de Bowen en tant qu'il semble être consubstantiel au genre romanesque ; il s'accompagne cependant aussi d'une subversion du genre littéraire, qui est une des visées du projet artistique de Bowen. Le descriptif participe ainsi dans ses romans d'une logique de l'intrusion dans la narration (ce en quoi il la subvertit au sens où Hamon l'entend), mais il est aussi constitutif d'une subversion du genre romanesque même. Bowen, qui mêle dans sa fiction les traditions victoriennes et modernistes ${ }^{3}$, y explore les limites de l'écriture dans sa tentative de sortir des canons langagiers du roman afin, à l'image de ses contemporains modernistes, de mieux refléter la réalité «telle qu'elle est vécue " ${ }^{4}$. Bowen utilise ainsi dans ses romans d'autres genres artistiques, ce qui permet de caractériser la subversion du descriptif à deux niveaux: d'une part à travers un double mouvement où les arts invoqués dans la 
description en subvertissent la littérarité en même temps qu'ils sont eux-mêmes subvertis à des fins littéraires dans ce processus. D'autre part, en ce que la mise en avant de leur caractère fragmentaire et de leurs faiblesses à représenter fidèlement le réel rejaillit sur la description, en mettant en cause sa nature mimétique.

L'idée selon laquelle le roman est un genre hétérogène est quasiment de l'ordre du lieu commun: il est universellement accepté qu'il est un mélange des genres qui l'ont historiquement précédé tels que la poésie ou le théâtre. Ceci se retrouve dans l'œuvre de Bowen, qui emploie largement le vocabulaire du théâtre, mais aussi celui des arts visuels : il pourrait ainsi sembler qu'elle s'inscrive dans certaines traditions du roman, comme celle de la genèse à partir du théâtre ou celle de l'Ut pictura poesis, du lien entre les deux «sister arts ». Toutefois, l'usage que fait Bowen des autres arts ne participe pas d'une démarche de transformation du roman en pièce de théâtre ou en tableau : elle semble au contraire mettre en avant ces deux genres artistiques pour mieux les détourner et utiliser le pouvoir représentationnel qui leur est propre afin de le mettre au service de la littérarité du roman.

Il n'est dès lors guère étonnant que ce soit à l'occasion des descriptions que soient utilisés d'autres genres artistiques; en effet, comme le constate Philippe Hamon dans Du descriptif , la description est souvent un lieu d'emprunt, où l'hétérogénéité du roman est à son apogée ${ }^{5}$. La transposition de ces considérations à l'œuvre de Bowen va nous permettre de montrer que la description y est le lieu d'une double subversion: une première subversion apparente du genre romanesque, rendue possible par l'usage d'autres genres artistiques, et une subversion de ces arts visuels et théâtraux pour les faire se plier aux exigences littéraires du roman.

5 Les descriptions d'ouverture de Bowen sont toujours d'une importance particulière : son côté post-victorien se manifeste ici, en ce qu'elle décrit de manière quasi-systématique la scène du roman ou les personnages avant que ne débute l'action. Dans The Heat of the Day ${ }^{6}$, qui est le roman que Bowen a écrit juste après avoir subi le «Blitz » de Londres et qui se déroule durant cette période, cette description d'ouverture se déroule dans un théâtre lors d'un concert, et les genres se mélangent immédiatement :

[...] at the start of the concert, this tarnished bosky theatre, in which no plays had been acted for some time, held a feeling of sequestration, of emptiness the music had not had time to fill. It was not completely in shadow - here and there blades of sunset crossed it, firing branches through which they travelled, and lay along ranks of chairs and faces and hands - gnats quivered, cigarette smoke dissolved. But the light was so low, so theatrical, and so yellow that it was evident it would soon be gone. The incoming tide was evening. Glass-clear darkness, in which each leaf was defined, already formed in the thicket behind the orchestra and was the other element of the stage (7)

6 Ce passage permet d'envisager comment sera utilisé le thème théâtral tout au long du roman. On peut tout d'abord observer l'usage ambigu du théâtre : le lieu de l'action est un théâtre détourné de son emploi principal, qui sert maintenant pour un concert musical, et non pour la représentation d'une pièce. Les pièces de théâtre sont un élément du passé, et c'est uniquement par la présence du bâtiment que ce passé continue à exister. Nous pourrions ainsi penser que le genre principalement exploré dans ce passage n'est pas le théâtre, mais la musique. En effet, elle est évoquée pour ses effets sur le psychisme des auditeurs : elle évoque des souvenirs et des émotions que les Londoniens ne connaissent plus en temps de guerre. Mais le théâtre a-t-il vraiment disparu? Le passage lui-même sème le doute : « the light was so low, so theatrical, and so yellow that it was evident that 
it would soon be gone ». Cette phrase peut sembler participer de la simple description du bâtiment: la lumière théâtrale (celle qui sort du théâtre tout autant que celle qui se caractérise par son essence théâtrale) est en train de disparaitre, sans doute au profit de la musique. Toutefois, une analyse plus approfondie permet de mettre en évidence un autre point de vue sur le théâtre: la théâtralité n'est plus contenue dans une structure architecturale, mais existe à l'extérieur de cette structure. Elle s'est "échappée » du bâtiment, et manifeste désormais sa présence de manière permanente dans la lumière du jour. Dans cette soirée d'été de 1942, la nature est devenue elle-même théâtrale. Les pièces de théâtre appartiennent donc peut-être au passé, mais le présent est vécu sur un mode théâtral, sans que les personnages puissent y échapper. En effet, la suite du passage met en question la possibilité que le bâtiment ait maintenant totalement perdu sa première fonction, car il émerge en quelque sorte de la lumière théâtrale : "It was not completely in shadow - here and there blades of sunset crossed it ». Enfin, l'ombre ellemême n'échappe pas à l'omniprésence de la théâtralité : « Glass-clear darkness, in which each leaf was defined, already formed in the thicket behind the orchestra and was the other element of the stage ".

Ce premier exemple illustre à quel point la subversion est au cœur même des descriptions de Bowen à travers l'emploi d'autres genres artistiques: la description en apparence anodine du théâtre déborde sur une vision elle-même théâtrale du cadre du roman. Cette subversion qui découle du mélange des genres est accentuée par un style où domine la contradiction et le paradoxe. En effet, le détail présenté dans une première phrase est rapidement contredit ou nuancé par la phrase qui suit. Ceci peut partiellement s'expliquer en raison des thèmes spécifiques traités dans ce roman. En effet, l'un des personnages de The Heat of the Day est un traître travaillant pour le compte des Allemands et, nous l'avons vu, le roman se situe à Londres durant la deuxième guerre mondiale : le mélange des genres témoigne ici de l'impossibilité à définir des frontières ou des limites dans un monde qui n'a plus de certitudes. Bien que The Heat of the Day en soit un exemple particulièrement flagrant, cette incertitude existe dans une certaine mesure dans tous les romans de Bowen : tous ses personnages se retrouvent sans repères à un moment ou un autre du récit.

8 L'omniprésence du théatre dans la description d'ouverture de The Heat of the Day se retrouve dans la première apparition de la protagoniste du roman, Stella, qui se déroule suivant une mise en scène élaborée :

Stella Rodney stood at the window of her flat, playing with the blind-cord. [...] Nothing is more demoralizing than waiting about for someone one does not wish to see. She mimed by this idiotic play at the window the disarray into which the prospect of Harrison had thrown her [...]. From the first he had shown her his imperviousness to everything she felt - would she be able to show him the indignity, if for himself only, of this impervious return? [...] In so far as she had set the scene at all, everything had been arranged to show that she did not care either for him, which he should know already, or as to anything more he might have to say. To show the careless negligence of her way of living, she had left the street door unlatched and the door of her flat, at the head of the stairs, ajar: thus it was left to him to make his own way in, unmet half way, without even that little taste of imperiousness to be got from the ringing of a bell, and with the best face he could. [...] Stella Rodney had taken this flat furnished, having given up the last of her own houses and stored her furniture when the war began. [...] Here in Weymouth Street she had the irritation of being surrounded by somebody else's irreproachable taste: the flat, redecorated in the last year of peace, still marked the point at which fashion in the matter had stood still - to those who were not to 
know this room was not her own it expressed her unexceptionably but wrongly.

(22-24)

Cette première description porte non pas sur les traits physiques de la protagoniste, mais sur ses actions : Stella attend la visite d'un homme dont elle a précédemment repoussé les avances. Cet homme, Harrison, s'apprête à lui dévoiler qu'il travaille pour le contreespionnage britannique. Il a découvert que Robert, l'amant de Stella, était en fait un traître, mais il ne le dénoncera pas si elle renonce à le voir et accepte de devenir sa maîtresse. Cette relation entre l'héroïne et son soupirant malvenu est placée sous le signe $\mathrm{du}$ théâtre (comme tout le roman d'ailleurs): ils ne communiquent pas (ou plus seulement) par la parole, mais par des gestes d'acteur : " She mimed by this idiotic play at the window the disarray into which the prospect of Harrison had thrown her ", "From the first he had shown her his imperviousness [...] would she be able to show him the indignity [...] of this impervious return? ». L'accent mis sur la nécessité de parvenir à montrer et non à dire évoque un cadre romanesque qui est justement contraires aux règles du roman luimême : il s'agit paradoxalement de la description d'une relation qui refuse de se décrire (c'est-à-dire d'utiliser le langage pour communiquer les détails), qui préfère la communication sans paroles. Les éléments que détaille cette description augurent de l'état de la relation qui relie Stella et Harrison. Les objets communiquent aussi un message sans langage, et participent ainsi à la mise en scène : "Insofar as she set the scene at all, everything had been arranged to show she did not care ». Toutefois l'emploi du mode théâtral n'est pas le simple fait de personnages "dramatiques » : la théâtralité découle aussi des conséquences de la guerre. Ainsi la scène que Stella s'apprête à représenter à Harrison n'est pas seulement destinée à lui transmettre un message (comme le montre le qualificatif «insofar»). Au contraire, le passage précise qu'une partie de cette mise en scène est exigée par les nécessités de l'époque : "Stella Rodney had taken this flat furnished, having given up the last of her own houses and stored her furniture when the war began [...] it expressed her unexceptionally but wrongly ». Le lieu même dans lequel se déroule le roman (Londres pendant la guerre) devient ainsi une scène théâtrale. Plus tard dans le roman, la description d'une vitrine d'épicier met l'accent sur ses cartons vides, qui symbolisent l'absence de nourriture en période de rationnement en temps de guerre : la nourriture réelle est remplacée par des accessoires de scène, la réalité par la théâtralité :

The main street was by now empty: today nothing more would happen. Before noon the housewives had swarmed, so completely, whitely, stripping the shops that one might ask oneself why these remained open. A scale or two adhered to the fishmonger's marble slab; the pastrycook's glass shelves showed a range of interesting crumbs; the fruiterer filled a longstanding void with fans of cardboard bananas and a 'Dig for Victory' placard; the greengrocer's crates had been emptied of all but earth by those who had somehow failed to dig hard enough. The butcher flaunted unknown joints of purplish meat in the confidence that these could not be bought; the dairy restricted itself to a china cow; the grocer, with costless courage, kept intact his stocks of dummy cartons and tins. In the confectioner's windows the ribbons bleached on dummy boxes of chocolate among flyblown cut-outs of pre-war blondes. Newsagents without newspapers gave out in angry red chalk that they had no matches either. Pasted inside a telephone booth, a notice asked one to telephone less. (72-73)

L'exemple de The Heat of the Day a sans doute une force particulière de par les thèmes qu'aborde le roman : la subversion des descriptions par le théâtre permet d'examiner les effets de la guerre sur les personnages. Mais nous ne saurions limiter l'usage du théâtre à 
sa présence thématique dans The Heat of the Day, la théâtralité n'est pas propre à ce roman, mais traverse toute l'œuvre de Bowen. L'auteur elle-même fournit une des raisons de son engouement pour la description théâtrale dans son article intitulé « Notes on Writing a Novel » :

The deadingness of straight and prolonged 'description' is as apparent with regard to scene as it is with regard to character. [...] For its details relevance is essential. Scene must, like the character, not fail to materialize.

[...] In 'setting a scene' the novelist directs, or attempts to direct, the reader's visual imagination. [...] the novelist must control his infatuation with his own visual power.

No not contributory image, must be the rule. Contributory to what? To the mood of the 'now', the mood that either projects or reflects action. It is a good main rule that objects - chairs, trees, glasses, mountains, cushions - introduced into the novel should be stage-properties, necessary for 'business'. It will be also recalled that the well-set stage shows many objects not actually necessary for 'business' but that these have a right to place by being descriptive - explanatory. In a play, the absence of the narrating voice makes it necessary to establish the class, period and general psychology of the characters by means of objects that can be seen. In the novel, such putting of objects to a descriptive (explanatory) use is excellent alternative to the narrator's voice. ${ }^{7}$

11 La description est ainsi constituée d'objets qui servent un effet de réel au sens de Barthes, mais la théâtralité existe surtout pour obliger le lecteur à considérer la description de la même manière qu'il aborde une scène de théâtre : la description peut paraître surchargée de détails et d'objets, mais, en réalité, elle est dépouillée de tout ce qui n'est pas utile, et c'est donc au lecteur de trouver l'intérêt, le sens, ou le symbolisme de chaque objet. La théâtralité de la description permet ainsi de rehausser sa littérarité : le fait même de subvertir le genre romanesque permet paradoxalement de l'enrichir.

Cette explication peut paraître paradoxale face au texte que nous venons d'analyser. Bowen y explique en effet que le but même de la description d'une scène est de présenter des détails, des objets, qui expriment des qualités essentielles à la compréhension du personnage auxquels ils appartiennent. Or, la description théâtrale que nous avons étudiée emploie des accessoires de scène qui n'expriment pas, ou mal, leur propriétaire, Stella. Stella est forcée malgré elle de jouer sur une scène qui subvertit les qualités essentiellement théâtrales des objets : ils n'existent pas pour exprimer sa personnalité, mais plutôt celle du propriétaire de l'appartement qu'elle occupe temporairement. Toutefois, l'emploi de ces vrais-faux accessoires de scène est en soi significatif. Le passé de Stella montre en effet qu'elle a toujours elle-même joué un rôle plutôt que de se rendre vulnérable à de vraies critiques : elle a par exemple laissé croire à son entourage qu'elle était la cause de l'échec de son mariage plutôt que d'avouer que son mari avait simplement trouvé quelqu'un d'autre. Le personnage de Stella est ainsi lui-même placé sous le signe de la représentation, ce qui résonne dans la nature des objets qui l'entourent.

La description théâtrale sert ainsi un but à la fois thématique et littéraire dans les romans de Bowen : The Heat of the Day en est une illustration. Elle permet tout d'abord d'explorer le thème des traumatismes engendrés par la guerre. À un second niveau, le fait de montrer plutôt que de dire peut sembler remettre en cause le genre romanesque auquel appartiennent ces descriptions. Toutefois, de la mise en cause du genre littéraire ressort une littérarité accrue, parce que la description «théâtrale " permet de mettre en avant 
pour le lecteur la pertinence des détails de la description pour la trame romanesque dans son ensemble.

Dans son texte sur l'importance de la description ci-dessus, Bowen fait également référence au visuel : «In setting a scene, the novelist directs, or attempts to direct, the reader's visual imagination. [...] the novelist must control his infatuation with his own visual power ». En effet, l'imagerie visuelle des romans de Bowen est extrêmement importante pour elle sur un plan personnel : elle s'est en effet tout d'abord destinée à la peinture avant de devenir écrivain, et sa pratique de l'écriture renvoie au processus d'élaboration d'une œuvre picturale : «Language is the writer's medium, used by him as the painter uses form, line, and colour $»^{8}$. Il n'est donc guère étonnant que Bowen emploie souvent ses descriptions à des fins d'hypotypose : elle souhaite "créer une image » dans l'esprit du lecteur. Toutefois, ses descriptions ne se limitent pas uniquement à des images fortes, ou encore à de simples descriptions picturales : elles s'apparentent souvent à de véritables tableaux. Ainsi, l'emploi des arts visuels participe également de la subversion du descriptif littéraire dans ses romans.

Dans Eva Trout par exemple, qui est le dernier roman de Bowen ${ }^{9}$, la description prosopographique $\mathrm{du}$ personnage de Constantine et les perceptions visuelles $\mathrm{du}$ personnage qui l'observe sont décrites dans un vocabulaire pictural :

The blond, massaged-looking flesh of Constantine's face seemed, like alabaster or indeed plastic, not quite opaque, having a pinkish underglow. It padded the bonestructure beneath it evenly - nowhere were there prominences or hollows or sags or ridges. The features, though cast in a shallow mould and severally unremarkable, almost anonymous, all the same were curiously distinct. What was strangest about them was, their relation to one another was for the greater part of the time unchanging: this was the least mobile face one might ever have seen. Now and then some few creases came into being, to supply their owner with such degree of expression as at that particular moment he chose to grant himself - or occasionally (though this was rarer) there was a calculated levitation of the eyebrows. Anything of that sort was, though, almost instantly wiped away.

Colour entered the picture, though used sparingly. Lips, for instance, were the naive fawn-pink of lips in a tinted drawing. Less perceptibly pencilled-in were the eyebrows, lashes, the exhausted pencil employed being gold-red. And the same tone reappeared in the hair; well nourished, though back from the forehead. And the eyes? These too were in the convention: a water-colourist's grey-blue. (36)

Dans un premier temps, le passage semble faire usage d'une métaphore plus proche de la sculpture que de la peinture : l'accent est mis sur le toucher, sur les matières. Toutefois, par la suite, la description est plus ouvertement assimilée à un tableau, et la matière et le toucher sont associés à des effets visuels comme ceux qui visent à donner une apparence de profondeur ou l'illusion d'une texture particulière, afin de garder une cohérence à la métaphore picturale: "Colour entered the picture [...] the naive fawn-pink of lips in a tinted drawing. Less perceptibly pencilled-in were the eyebrows, lashes, the exhausted pencil employed being gold-red. [...] And the eyes? [...] a water-colourist's grey-blue ».

Bien que la description s'éloigne délibérément et ouvertement du réalisme (le personnage y est décrit comme un objet fabriqué, et non comme un être humain que caractérisent ses traits physiques), le narrateur n'abandonne pas pour autant ses visées mimétiques. Au contraire, l'ekphrasis est ici particulièrement forte: la description des couleurs de l'aquarelle ou des crayons est tellement précise que le lecteur peut avoir l'impression de les voir. Ainsi, la couleur est réelle, même si le personnage ne l'est pas. L'emploi d'une 
description picturale permet donc à Bowen d'accroître et de diminuer simultanément la mimésis du passage.

La comparaison, voire le parallèle entre la description romanesque et le tableau se retrouve dans la quasi-totalité des romans de Bowen. Ainsi dans The Hotel ${ }^{10}$, par exemple, les couchers de soleil sur la Riviera italienne sont décrits comme s'ils étaient peints sur une toile, ce qui renvoie aux tableaux, bien concrets ceux-là, qu'en font les touristes: "There were about seven ladies present, all embroidering something unpractical and therefore permissible, except Mrs. Duperrier, who, poor soul, was too restless, and another lady who with the aid of much gamboge and vermillion was touching up a watercolour, a sunset. » (52). Le lien entre la peinture pratiquée par les personnages (au sein de la trame romanesque) et la peinture, prise au sens métaphorique, est renforcé par les commentaires de la protagoniste du roman : " If I were Monet and alive now, I would paint this and present the picture to the P.L.M., as a poster for the Côte d'Azur » (55). De même, l'omniprésence des tableaux se manifeste également dans les pensées des personnages rapportées par le narrateur. À trois reprises, deux personnages différents de The Hotel imaginent leurs actions comme le sujet d'un tableau : ainsi le paysage est décrit par une des personnages comme le sujet d'un tableau intitulé «Flight into Egypt » (34), les personnages devant le paysage deviennent un tableau intitulé "English Visitors" (83), et l'agacement que provoquent d'autres touristes évoque l'image d'un tableau qui serait intitulé « Temptation to Murder » (109).

Toutefois, dans les romans de Bowen, l'accent n'est pas seulement mis sur les descriptions qui se rapprochent de tableaux; la description de véritables tableaux y est également chose courante. En règle générale, il ne s'agit pas de tableaux réellement existants, mais de tableaux représentant des personnages et des scènes du roman. Cet usage sert à étendre la signification du genre pictural dans les descriptions. Ainsi, dans The Little Girls ${ }^{11}$ , les petites filles éponymes sont fascinées par une vitrine dans laquelle se trouvent des tableaux qui dépeignent la réalité qui les entoure :

This was an old High Street window not to be missed: it guaranteed a sensation like no other - that of looking at pictures of where one was. The back of the window built itself up with masterpieces, among them 'Hope', still clutching her harp, but the front showed water colours and etchings of the Old High Street [...] But the greater wonder was that, outside the pictures, there the Old High Street actually was. You could verify simply by turning round: there, it indeed remained - a magnified picture.

So seeing it, one saw it for the first time... Moreover, a portion of the old High Street (that exactly across the way from the picture shop) reflected itself not only in the shop window but in the glass of these numerous pictures of itself. The reflection itself looked like a large painting.

The gables, etc., were there twice over. (97-98)

Cette description du tableau est particulière en ce qu'il déborde de son cadre. En effet, il est vu comme inséparable de son sujet d'inspiration: la description est donc de fait celle de la relation entre le tableau décrit et la vie qui l'inspire et qu'il inspire (ou du moins, affecte) à son tour. L'art visuel est ici en quelque sorte une mise en abyme de l'art romanesque. La discussion du lien entre le tableau et l'endroit qu'il dépeint, et le dédoublement qui se fait par (ou sous) la glace des cadres et de la vitrine, visent à mettre en avant l'idée que les deux arts (littérature et tableau) sont en fait les mêmes : si la partie de Old High Street qui est en face du magasin se reflète dans la glace et a elle-même l'air d'être peinte, c'est qu'elle l'est. Bowen s'appuie ainsi sur l'importance du visuel dans son roman pour montrer qu'il est lui-même un tableau. Comme le théâtre, l'art visuel est 
donc omniprésent dans le roman de Bowen : ici le tableau de High Street et la réalité créée par l'auteur sont pour ainsi dire identiques. L'intérêt de ce procédé est mentionné dans la description elle-même: "So seeing it, one saw it for the first time...». Cette phrase a deux sens possibles : d'une part, utiliser des tableaux comme une mise en abyme du roman effectue une sorte de défamiliarisation, et permet de redécouvrir, et le roman, et la description. Cette défamiliarisation permettrait donc à la description de reprendre l'importance qu'elle perd souvent aux yeux du lecteur peu attentif. Mais, d'autre part, le lecteur n'est pas comme les personnages : il « voit » cette rue pour la première fois. Si l'on interprète donc cette phrase du point de vue du lecteur, l'accent n'est alors plus mis sur le fait de revoir, mais de voir tout court : l'usage des arts visuels dans les textes de Bowen oblige le lecteur à voir, à entrer plus profondément dans l'histoire parce qu'il doit la visualiser. L'ekphrasis rejoint ainsi l'hypotypose.

21 Ainsi, nous pouvons considérer que les autres genres sont en fait à la fois une subversion et une mise en abyme du genre romanesque : l'art visuel permet d'une part de montrer les limitations du langage (ne pas pouvoir "voir») et de l'autre d'essayer de combler ce manque par des descriptions élaborées. L'usage de procédés qui s'appuient sur le visuel dans les descriptions de Bowen oblige ainsi le lecteur à les visualiser. De même, la mise en abyme permet de reconnaître que les personnages et le monde qu'ils habitent sont comme les tableaux qu'ils admirent : ils font eux aussi partie d'une œuvre artistique.

L'importance particulière du tableau est aussi due à son emplacement au cœur des descriptions : il permet, tout comme nous l'avons montré pour l'emploi du genre théâtral, de mettre en avant la description en tant que telle. La description, rappelons-le, est déjà dans une certaine mesure une subversion du genre narratif, puisqu'elle interrompt la cadence de la narration et de l'action pour obliger le lecteur à passer du temps à réfléchir sur les détails d'un visage, d'un paysage, etc. Le tableau, genre de l'espace et non du temps comme l'est la littérature, met en avant cette rupture de la temporalité effectuée par la description. Cette subversion du genre littéraire par les arts visuels s'avère donc être une fois de plus une mise en avant de la littérarité du texte : l'emploi d'un autre art permet de privilégier les qualités particulières de la description, et ainsi de rehausser la valeur artistique du roman, puisqu'il en fait un tout plus cohérent; la description n'est donc pas accessoire, mais constitue ainsi au contraire l'essence même de l'intérêt du roman.

Ce n'est donc pas un hasard si Bowen parle du théâtre et des arts visuels dans ses commentaires sur l'importance des descriptions. En effet, les deux arts remplissent la même fonction dans ses romans, sous des modalités qui leur sont propres. Bien qu'en apparence subversifs pour la " pureté » romanesque de la description, ils s'avèrent donc être eux-mêmes subvertis à des fins purement littéraires : le théâtre comme l'art visuel sont tous les deux des subversions en même temps que des mises en abyme de l'art littéraire, et permettent, chacun à leur manière, de le mettre en lumière.

Les arts visuels et théâtraux ne sont cependant pas toujours présentés sous un jour entièrement positif. Au contraire, ils sont souvent considérés comme fragmentaires, limités, et imparfaits dans leur qualité d'arts de la représentation. Puisque ces deux arts sont des mises en abyme du genre romanesque lui-même, puisqu'ils servent à accentuer la littérarité de la description, il semble possible que cette deuxième subversion signifie une mise en cause de la littérature romanesque. C'est pour examiner cette possibilité qu'il est nécessaire de reprendre successivement l'emploi des deux arts dans les textes de Bowen. 
theâtre, nous l'avons vu, permet à Bowen d'obliger le lecteur à regarder les détails des descriptions de plus près, puisque les objets qui y sont décrits sont de véritables accessoires de scène, primordiaux pour la compréhension de la suite de l'intrigue. Toutefois, les allusions à l'art théatral sont tout aussi souvent synonymes d'artifice. Ainsi par exemple dans The Death of the Heart ${ }^{12}$, l'insistance sur le théatre est omniprésente, mais l'accent est surtout mis sur la tromperie implicite dans la scène. Le paysage londonien et la maison dans laquelle habitent les personnages principaux sont décrits dans un premier temps de façon mimétique et réaliste, mais ils s'avèrent par la suite n'être qu'un décor, supprimant ainsi le caractère mimétique de la description :

As though he [Thomas] heard himself challenged, or heard an echo, he looked sharply over his shoulder down the terrace - empty, stagy, E-shaped, with frigid pillars cut out on black shadow; a façade with no back. (44)

At the far side of the road, dusk set the Regency buildings back at a false distance: against the sky they were colourless silhouettes, insipidly ornate, brittle, and cold. The blackness of the windows not yet lit or curtained made the hollows look hollow inside. (12)

La scène décrite dans les deux citations est le lieu principal où se déroule l'action du roman. Qu'elle soit ici traitée comme une scène de théâtre ne fait pas de doute : le lexique en témoigne («stagy », « the windows not yet lit or curtained»), tout comme le fait qu'il s'agisse non d'un décor naturel, mais du produit d'une fabrication, avec des matériaux et des lumières qui donnent une impression de profondeur («E-shaped with frigid pillars cut out on black shadow; a façade with no back », « dusk set the Regency buildings back at a false distance »). Ce lexique nous présente aussi sous un jour péjoratif cette nouvelle facette de l'art théâtral : par analogie, le théâtre est lui aussi superficiel, vide, insipide, sans couleur, froid, creux, ou encore tout simplement faux. Le fait que la maison soit décrite aussi ouvertement comme un décor, et que cette description insiste non sur ses vertus esthétiques, mais uniquement sur son aspect superficiel et faux, constitue une véritable mise en cause de cet art et, de fait, de son usage romanesque dans les descriptions.

Cette mise en cause ne se limite pas au simple décor : en effet, les personnages sont euxmêmes présentés comme des comédiens. Ainsi dans The Death of the Heart, la relation entre Eddie, (un jeune homme décrit à la fois comme ambitieux, paresseux, et cupide), et Anna, la femme de son patron, qu'il méprise et admire tour à tour, est décrite comme une scène théâtrale, comme par exemple quand elle lui propose de travailler dans l'entreprise de son mari. La réaction d'Eddie, qui interprète cette offre comme une tentative d'Anna de s'éloigner de lui, témoigne bien de la nature théatrale de leur relation :

Eddie turned away and stood looking out of the window at the park. Shoulders squared, hands thrust in his pockets he took the pose of a chap making a new start. Her aquamarine curtains, looped high up over his head with cords and tassels, fell in stately folds each side of him to the floor, theatrically framing his back view. He saw the world at its most sheltered and gay: it was, then, the spring of the year before; the chestnuts opposite her window were in bud; through the branches glittered the lake, with swans and one running dark pink sail; the whole scene was varnished with spring light.

Eddie brought one hand out of his pocket and pinched a heavy moiré fold of the curtain by which he stood. This half-conscious act was hostile: Anna heard the moiré creak between his finger and thumb. She did not for a moment doubt that in his own mind Eddie was travestying the scene. (69-70) 
un premier temps, ce sont les ressemblances avec The Heat of the Day qui prévalent dans le traitement du théâtre: le thème est apparent dans la présentation du monde romanesque lui-même, à travers la mention des rideaux (" theatrically framing his back view ») et du paysage qui est le cadre du décor ("the whole scene was varnished with spring light »). De même, tout comme Stella dans The Heat of the Day, Eddie agit ici en comédien : "Shoulders squared, hands thrust into pockets he took the pose of a chap making a new start ». Il tourne le dos à la réalité pour faire face à la scène, aux rideaux du théâtre, ce qui traduit son refus de quitter son masque, son rôle. Toutefois, il est important de noter qu'Eddie se conforme à ce qu'Anna attend de lui, tout en parodiant ses attentes. En effet, Eddie met en avant la théâtralité de la "réalité ", où pour réussir à vivre en société il faut savoir jouer son rôle. Il souligne la présence des artifices traditionnels qu'emploie le théâtre («Eddie brought one hand out of his pocket and pinched a heavy moiré fold of the curtain by which he stood. This half-conscious act was hostile: Anna heard the moiré creak between his finger and thumb »). Ainsi, la mise en scène d'Eddie montre la fausseté de cette représentation que constituent ses actions, ses réactions et celles d'Anna: ce qui est censé être "réel» n'est, en réalité, qu'illusion théâtrale. Les deux personnages savent que la signification de cette offre d'emploi dépasse celle qu'ils laissent paraître dans leur dialogue : elle implique un changement profond dans leur relation. De cette façon, l'outrance du jeu d'Eddie et son insistance sur la théâtralité de son personnage mettent en relief la fausseté qu'impliquent les conventions de la bienséance : "She did not for a moment doubt that in his own mind Eddie was travestying the scene ». Paradoxalement, l'usage de la métaphore théâtrale devient une façon pour le personnage de dénoncer la théâtralité (et par extension la fausseté) de la société. L'usage du théâtre n'est plus une façon de donner un sens nouveau au roman, mais une manière de souligner l'omniprésence du paraître qui cache le sens profond des actions.

Le paradoxe central de cet usage du théâtre réside dans le fait que c'est effectivement le narrateur qui met en scène, qui joue le rôle de dramaturge et de réalisateur. De ce point de vue le théâtre est bel et bien une mise en abyme du roman. L'artifice du théâtre est ainsi le pendant de l'artifice du roman, qui est employé afin de représenter la réalité, mais n'est en fait qu'un faux-semblant.

subit lui aussi une mise en cause de ses qualités représentationnelles. Ainsi, dans notre exemple tiré de The Little Girls où le tableau dédouble la réalité, ce tableau est loin d'être une réussite totale, et sa qualité en tant que représentation est mise en doute, ce que montre l'analyse de l'intégralité du passage :

This was an Old High Street window not to be missed: it guaranteed a sensation like no other - that of looking at pictures of where one was. The back of the window built itself up with masterpieces, among them 'Hope', still clutching her harp, but the front showed water colours and etchings of the Old High Street - far more queer, as a street, than one had thought. Top parts of buildings stuck out, hatted by too-large gables, black beams squeezed doors, windows and archways out of shape. Nothing high but the crooked chimneys. Toy shop-fronts were frowned over by dimmed sign-boards, from under which they looked ready to fall forward... All this was to be wondered at through two layers of glass - the picture - shop's windows and the glass in the gilt or ebony frames. (97-98, c'est moi qui souligne)

La réalité telle qu'elle est représentée par le tableau s'inscrit dans la tradition dickensienne : le paysage est personnifié ou animé par des sentiments («top parts of buildings [were] hatted by too-large gables », "the toy-shops were frowned over by 
dimmed sign-boards»). Il est aussi architecturalement impossible, prêt à tomber en ruine, totalement disproportionné. La réalité que propose le tableau n'est donc pas celle d'une description mimétique : le réalisme, qui serait normalement le but d'un tableau qui souhaite retranscrire en forme picturale, est ici loin d'être atteint. Le tableau est ainsi mis en cause dans ce que nous avons considéré comme son but principal, qui est de faire visualiser le lecteur. Ici le lecteur est mis en face de cette tentative de la part de l'artiste du tableau de dépeindre visuellement la scène, mais celle-ci est clairement un échec : le lecteur ne peut visualiser que la version peinte de la réalité, une version grotesque du monde romanesque toujours considéré comme essentiellement vraisemblable et réaliste. Le fait que le narrateur du roman ne montre que le tableau imparfait du lieu, et non le lieu lui-même, implique que cette distorsion est la seule version de la réalité du monde romanesque disponible pour le lecteur. Le roman dans sa qualité de représentation est ainsi lui aussi mis en cause.

Un tel échec s'inscrit partiellement dans la visée souvent comique des romans de Bowen, mais il reflète surtout une critique de la vraisemblance du genre pictural. En effet, dans les romans de Bowen, l'accent est souvent mis sur la qualité nécessairement artificielle et partielle de la représentation en deux dimensions : cet aplatissement de l'image (comme l'aplatissement du globe sur un planisphère) ne peut pas se faire sans distorsion. Ainsi dans Friends and Relations ${ }^{13}$, quand les deux protagonistes, qui sont sœurs, se rendent compte qu'elles sont toutes les deux amoureuses du même homme, l'image que chaque sœur a de l'autre est décrite comme un tableau déformé :

These weeks, a grotesque, not quite impossible figure, had come to interpose between herself and Laurel. [...]

She resembled each for the other, and pressing in between them since they had permitted themselves to part a little interposed a preposterous profile that to each, at the very edge of her vision, was somehow darkly familiar. [...] This ever-presence in profile had, for each of the sisters, the Egyptian effective defect: from Janet's side or from Laurel's - could either have seen her, she was so close, or, faced her, she was so dreadful - two eyes were visible, forced elsewhere with an undeviating intentness. The look directed upon Edward with its whole darkness. (122)

Les images respectives de chaque sœur ne correspondent pas à une représentation fidèle de leur personne, mais à un simple tableau, qui effectue le passage de trois dimensions à deux. L'image qui en résulte est distordue: le narrateur utilise ici la métaphore des procédés utilisés dans la peinture égyptienne, où les figures sont un mélange de plusieurs perspectives (dans l'art égyptien le visage est vu de profil, l'œil de face, le torse de face, le bassin de trois quarts, et les jambes de profil et décalées ${ }^{14}$ ). Chaque sœur ne voit plus que les yeux de sa rivale, qui n'est alors plus tout à fait sa sœur. L'image en deux dimensions est ainsi une image faussée, partielle, qui ne correspond plus à la réalité de la personne dépeinte. Dans To The North ${ }^{15}$, la brève description de la relation des deux personnages qui vivent sous le même toit confirme la distorsion qu'implique le tableau: "In their life together, as in a quiet marriage, Emmeline and Cecilia, inquiring less and less, each affectionately confronted the other's portrait of her own painting, finding it near enough to reality » (148). Là aussi, l'image partielle de l'autre est décrite en termes picturaux : elle se rapproche d'une représentation fidèle sans pour autant y arriver. Le tableau, chez Bowen, se prête ainsi à une compréhension partielle et superficielle des personnages.

Afin de dépasser la distorsion du tableau qui résulte de la transposition d'une réalité en trois dimensions et deux dimensions, Bowen adopte un point de vue proche de celui des cubistes, au sens où elle reprend le principe d'une représentation simultanée des trois 
dimensions dans le tableau. Les descriptions qui utilisent des procédés cubistes sont d'ailleurs nombreuses dans les romans de Bowen : ainsi dans The World of Love, To the North , et The Hotel, l'apparence des personnages se compose de fragments d'images en deux dimensions collées les unes aux autres pour voir de plusieurs points de vue. De même, dans The Heat of the Day, Harrison est décrit comme ayant un œil plus haut que l'autre, ce qui rappelle certains personnages de Picasso. Toutefois, ces tentatives de rendre les trois dimensions de la réalité dans les "tableaux » décrits dans les romans s'avèrent être des échecs; la fragmentation en est toujours le résultat. L'art visuel semble donc perdre sa qualité d'hypotypose par la distorsion et la fragmentation que Bowen met en avant dans ses romans.

Cette subversion des qualités représentationnelles des arts semble contredire notre constat de la subversion de ces arts à des fins littéraires: alors que les arts théâtraux et visuels sont utilisés par Bowen pour accentuer la littérarité du texte et pour mieux situer l'importance de la description, ces mêmes arts sont par la suite vus comme fragmentaires, limités, dans l'impossibilité de représenter la réalité de façon convaincante. Chaque art a ses propres techniques pour représenter la réalité, mais ces techniques, accentuées dans les textes de Bowen, brisent la bienséance littéraire dont le lecteur a l'habitude, et le rendent encore plus conscient qu'elles ne sont que des artifices. Ainsi, en exploitant et dévoilant simultanément les techniques employées par le théâtre et la peinture, Bowen met en avant les limitations de son propre art, qui ne peut prétendre apporter la réalité, mais uniquement en présenter un faux-semblant. Cette mise en cause des arts est un aveu que la représentation reste limitée, mais traduit aussi un refus de se cacher derrière les subterfuges d'un réalisme naïf, ce en quoi Bowen se place résolument dans le camp du modernisme.

Ainsi, la vraie subversion du descriptif réside dans sa dualité, et dans la position paradoxale qu'elle occupe dans l'œuvre de Bowen. En effet, Bowen cherche à mettre en avant la richesse de son art à travers les métaphores des arts visuels et théâtraux, mais sans pour autant oublier d'attirer l'attention du lecteur sur les faiblesses et les échecs de ce même art. Ses descriptions constituent donc une clé pour comprendre la dualité de son œuvre: mi-victorienne, mi-moderniste, elle cherche à représenter la réalité de façon convaincante, mais sans pour autant cacher les limites de ce qui n'est jamais qu'une représentation.

\section{NOTES}

1. «De fait, sorte de champ vacant, ou de degré zéro méthodologique ( $c f$. les couples canoniques : descriptif $v s$ narratif, descriptif $v s$ interprétatif, descriptif vs performatif, etc.), le descriptif ne semble être jamais qu'un lieu ou moment transitoire pour passer à de plus nobles objets d'étude. » Philippe Hamon, Du descriptif, Paris : Hachette, 1993, 6-7.

2. "L'essence du descriptif, s'il devait en avoir une, son effet, serait dans un effort: un effort pour résister à la linéarité contraignante du texte [...] »ibid., 5. 
3. En effet, chronologiquement Bowen écrit à la fin de l'époque moderniste, et elle emploie des techniques typiques de ce mouvement. Toutefois, ses expérimentations littéraires, qui s'apparentent au modernisme, s'accompagnent de certains éléments plutôt caractéristiques d'un roman réaliste, comme par exemple l'usage d'un narrateur omniscient qui présente et commente le monde du roman.

4. C'est sans doute Virginia Woolf dans ses célèbres commentaires sur le roman qui explicite le mieux la conception du réel que prônent les modernistes : "The mind, exposed to the ordinary course of life, receives upon its surface a myriad impressions - trivial, fantastic, evanescent, or engraved with the sharpness of steel. From all sides they come, an incessant shower of innumerable atoms, composing in their sum what we might venture to call life itself; and to figure further as the semi-transparent envelope, or luminous halo, surrounding us from the beginning of consciousness to the end. Is it not perhaps the chief task of the novelist to convey this incessantly varying spirit with whatever stress or sudden deviation it may display, and as little admixture of the alien and external as possible? We are not pleading merely for courage and sincerity; but suggesting that the proper stuff for fiction is a little other than custom would have us believe it. » Virginia Woolf, « Modern Novels », The Essays of Virginia Woolf: vol. 3 1919-1924, Andrew McNeillie (ed.), San Diego: Harcourt Brace Jovanovich, 1988, 33.

5. "Notons d'abord qu'un savoir (de mots, de choses) est non seulement un texte déjà appris, mais aussi un texte déjà écrit ailleurs, et la description peut donc être considérée toujours, peu ou prou, comme le lieu d'une réécriture, comme un opérateur d'intertextualité; de-scribere, rappelons-le, étymologiquement, c'est écrire d'après un modèle ", Philippe Hamon, Du descriptif, op. cit., 48.

6. Elizabeth Bowen, The Heat of the Day (1948), London: Penguin Books, 1976.

7. Elizabeth Bowen, « Notes on Writing a Novel », The Mulberry Tree, Hermione Lee (ed.), San Diego CA: Harcourt Brace Jovanovich, 1986, 40-48.

8. Elizabeth Bowen, Seven Winters and Afterthought (1950), New York: Alfred A. Knopf, 1962, 92.

9. Elizabeth Bowen, Eva Trout, London: Penguin Books, 1968.

10. Elizabeth Bowen, The Hotel (1927), London: Penguin Books, 1956.

11. Elizabeth Bowen, The Little Girls (1963), London: Penguin Books, 1982.

12. Elizabeth Bowen, The Death of the Heart (1938), London: Penguin Books, 1966.

13. Elizabeth Bowen, Friends and Relations (1931), London: Penguin Books, 1959.

14. "Art égyptien », Encyclopédie de l'art, Lucio Felici (dir. de rédaction), François Livi (trad.), Paris : Livre de Poche, 1991, 333.

15. Elizabeth Bowen, To the North (1932), London: Penguin Books, 1960.

\section{INDEX}

oeuvrecitee Heat of the Day (The), Death of the Heart (The), Eva Trout, Hotel (The), Little Girls (The), Friends and Relations, To the North, World of Love (The) 